\title{
Ultralow Threshold On-Chip Toroidal Microcavity Nanocrystal Quantum Dot Lasers
}

\author{
Bumki Min ${ }^{1}$, Sungjee Kim ${ }^{1}$, Koichi Okamoto ${ }^{2}$, Lan $\operatorname{Yang}^{1}$, Axel Scherer ${ }^{1,2}$, Harry Atwater ${ }^{1}$, and \\ Kerry Vahala ${ }^{1}$ \\ ${ }^{1}$ Department of Applied Physics, California Institute of Technology, Pasadena CA 91125 \\ ${ }^{2}$ Department of Physics, California Institute of Technology, Pasadena CA 91125 \\ bkmin@caltech.edu
}

\begin{abstract}
We demonstrate an on-chip toroidal microcavity nanocrystal quantum dot laser with a threshold energy below 10 femto-Joules at room temperature, a factor of 1 million lower than previously reported for strongly-confined, nanocrystal quantum dot lasers.

(C)2006 Optical Society of America

OCIS codes: (140.4780) Optical resonators; (140.5960) Semiconductor lasers
\end{abstract}

Reduction of laser threshold can be obtained by leveraging large oscillator strength gain media with modern, highquality-factor/high-spatial-confinement resonator designs. Along these lines, two, emerging strategies have been pursued as a means to explore the limits of classical laser performance. In the first, high spatial confinement photonic crystal microcavities offering moderate Q factors are loaded with epitaxially-grown, self-assembled semiconductor quantum dots (QDs) with impressive results [1]. In the latter, dielectric microsphere resonators providing high-Q performance with moderate spatial confinement have been coated with strongly-quantum-confined, semiconductor colloidal dots to also achieve excellent performance [2]. As a chip-based microcavity providing Q factors in excess of 100 million, silica toroidal microcavity resonators provide both long photon storage times and improved spatial confinement over microsphere resonators of comparable size [3]. The devices are also fabricated at lithographically pre-defined locations on a silicon chip which provides additional benefits over microsphere resonators. Furthermore, with highly-ideal, tapered-fiber coupling, efficient pumping and laser emission extraction is possible.

Ultrahigh-Q silica toroidal microcavities and tapered fiber waveguides are fabricated by the procedures published earlier [3]. CdSe/ZnS (Core/Shell) QDs of high photoluminescence (PL) quantum yield (> 50\%) are similarly synthesized as published previously [4]. The QDs are dispersed in toluene and spin-cast on toroidal microcavities. Thin, densely-packed QD films are generally formed on the surfaces of the toroidal microcavities. The thickness can be tuned down to the submonolayer by controlling the spin-casting condition and the concentration of the QD solutions. Typical QD thicknesses explored in this work were a few densely-packed layers down to $\sim 0.3 \%$ (sub-monolayer) surface coverage. Figure la shows the schematic experimental set up. Pump pulses are coupled to the input fiber end and delivered to the toroidal microcavity. The lasing emission is bidirectionally coupled out of the cavity as shown in the optical micrograph (inset of Fig. 1a) and is measured at one end of the tapered fiber either by a streak camera with time resolution of $20 \mathrm{ps}$ or by a liquid nitrogen cooled CCD spectrometer.

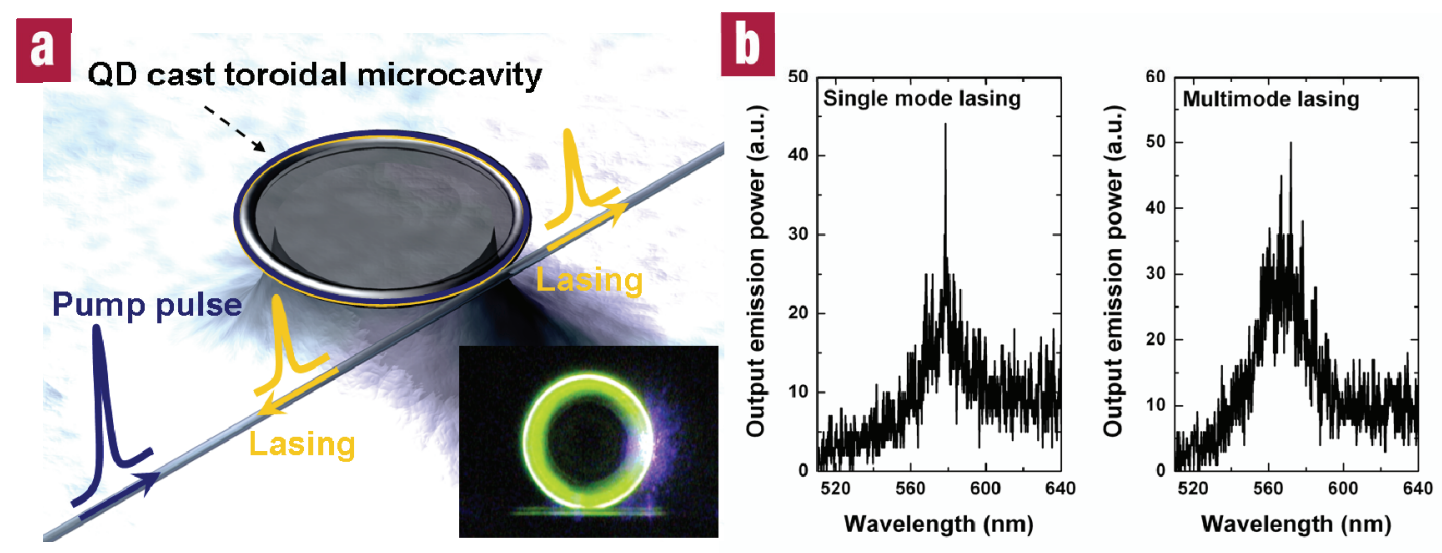

Fig. 1. (a) Schematic experimental setup of a tapered-fiber-coupled, toroidal-microcavity, nanocrystal QD laser. Inset: Optical micrograph of a tapered-fiber-coupled, toroidal-microcavity, nanocrystal QD laser. (b) Single mode and multimode lasing spectra. 


\section{QWD6.pdf}

Pulsed excitation is used for all subsequent experiments. The pulse is frequency doubled to $388 \mathrm{~nm}$ from a mode-locked Ti:sapphaire laser having a pulse width of $<100 \mathrm{fs}$ and a repetition rate of $80 \mathrm{MHz}$. Optimal pump coupling resulted when tapered fiber and toroidal microcavity were in contact. This arrangement clearly indicates the presence of strong pump-band absorption provided by the QDs. Based on measured pump coupling (10 - $30 \%)$ and estimated pump Q factor, the waveguide-resonator loading is believed to be in the under-coupled regime.

Depending on the sample, both single-mode and multimode lasing have been observed for tapered fiber coupled QD cast toroidal microcavities. Representative single mode lasing is shown in Fig. 1b (left panel) with the line narrowed lasing peak at $579 \mathrm{~nm}$. Figure $1 \mathrm{~b}$ (right panel) shows a typical multimode lasing spectrum. In this sample, the estimated FSR, based on the measured principal diameter of the toroidal cavity, is about $1 \mathrm{~nm}$, whereas the lasing peaks are separated by about $5 \mathrm{~nm}$. The absence of next-nearest-neighbor longitudinal modes in this spectrum suggests that spectral hole burning (SHB) is suppressing the oscillation of these modes. The expected SHB spectral width matches well with the observed peak separations [5].

Figure 2a shows typical L-L curves measured using a tapered-fiber coupled QD laser that has 200,000 QDs within the active region. The saturation induced by multiexciton generation is observed at higher excitation intensity (left panel). Two optical micrographs corresponding to the saturation regime and the lasing onset are shown as insets in the figure. As can be seen from the plot in the right panel of Figure 2a, magnified near the onset of threshold, a threshold energy $(\sim 1 \mathrm{pJ})$ is measured. We next studied the effect of reducing the number of QDs on the threshold. To test for an optimum threshold, the output emission power is plotted as a function of input pump energy for two other samples having a decreased number of QDs (Fig. 2b). Threshold pump energies of $42.4 \mathrm{fJ}$ and $9.9 \mathrm{fJ}$ are obtained for toroidal microcavities that have $\sim 20,000$ and $\sim 2,000$ QDs, respectively within the active region. The measured threshold energy of $9.9 \mathrm{fJ}$ is over one million times smaller than previously reported results using nanocrystals. For comparison, the same two L-L curves are presented in a magnified view in the right panel of Fig. $2 b$.

These observed improvements in laser threshold demonstrate the effectiveness of colloidal QDs as a tool to explore the limits of laser performance in the strongly confined regime, and, in addition, the utility of tapered fiber coupling. The observed threshold trends with decreasing QD number confirm theoretical trends resulting from transparency. In addition, from a fundamental viewpoint, the measurement of $9.9 \mathrm{fJ}$ is a record low threshold for strongly-confined QDs and is close to the packing fraction bound for operation in this regime [6].
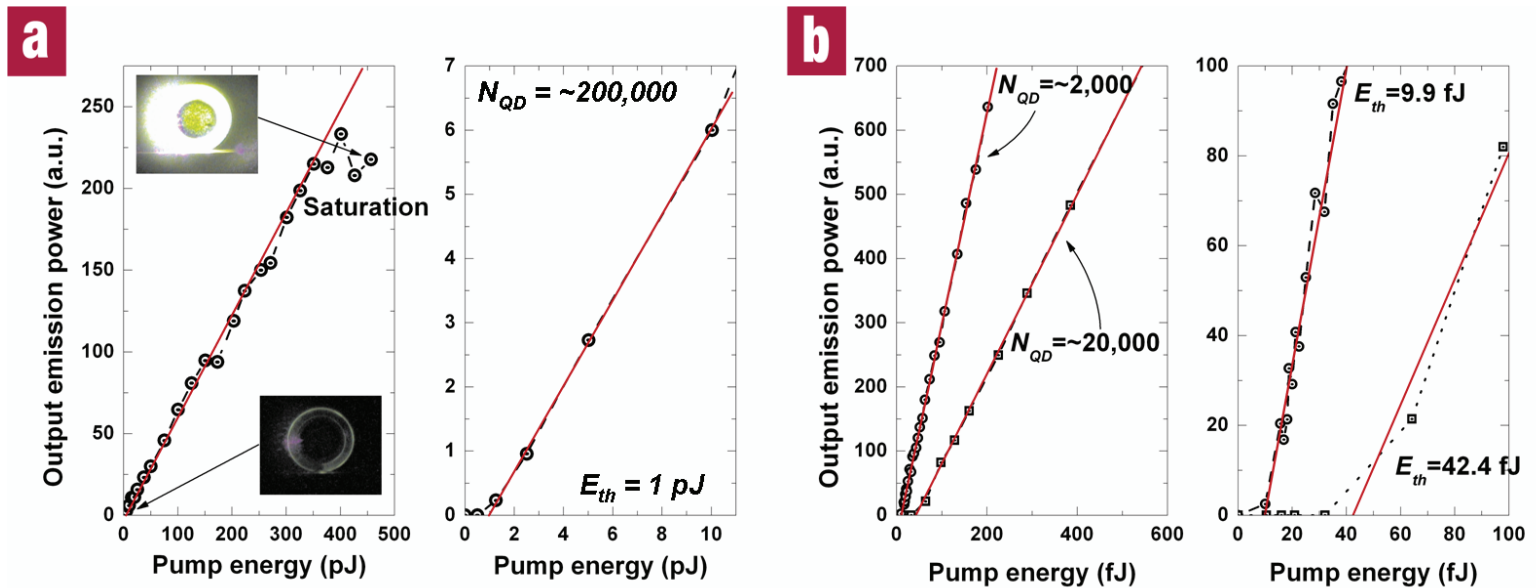

Fig. 2. (a) L-L curves from toroidal microcavity QD lasers excited via tapered-fiber coupling. Output emission power plotted as a function of input pump energy at the tapered-fiber microcavity junction. (b) Output emission power plotted as a function of input pump energy for two samples with decreased number of QDs in the active gain region. For a sample with $\sim 2,000$ QDs in the active region, an ultralow threshold energy of $9.9 \mathrm{fJ}$ is observed. For comparison, the L-L data curves are repeated (right panel) with a magnified pump/emission scaling (slope efficiencies are arbitrarily chosen for clarity).

\section{References}

[1] S. Strauf et al., "Photonic crystal quantum-dot laser with ultra-low threshold," in proceedings of CLEO/QELS 2005, paper QWA1 (2005).

[2] P. T. Snee et al., "Whispering-gallery-mode lasing from a semiconductor nanocrystal/microsphere resonator composite," Adv. Mater. 17, 1131-1136 (2005)

[3] D. K. Armani et al., "Ultra-high- $Q$ toroid microcavity on a chip," Nature 421, 925-928 (2003).

[4] M. A. Hines et al., "Synthesis and characterization of strongly luminescing ZnS-capped CdSe nanocrystals," J. Phys. Chem. 100, 468-471 (1996).

[5] M. Shim et al., "Intraband hole burning of colloidal quantum dots," Phys. Rev. B 64, 245342 (2001).

[6] V. I. Klimov et al., "Optical gain and stimulated emission in nanocrystal quantum dots," Science 290, 314-317 (2000). 